\title{
Surface-mediated attraction between colloids
}

\author{
H. Kaïdi, ${ }^{1}$ T. Bickel, ${ }^{2}$, ${ }^{\text {and }}$ M. Benhamou ${ }^{1}$ \\ ${ }^{1}$ Laboratoire de Physique des Polymères et Phénomènes Critiques, \\ Faculté des Sciences Ben M'sik, B.P. 7955, Casablanca, Morocco \\ ${ }^{2}$ Centre de Physique Moléculaire Optique et Hertzienne, Université Bordeaux 1, \\ 351 cours de la Libration, 33405 Talence, France
}

(Dated: November 25, 2018)

\begin{abstract}
We investigate the equilibrium properties of a colloidal solution in contact with a soft interface. As a result of symmetry breaking, surface effects are generally prevailing in confined colloidal systems. In this Letter, particular emphasis is given to surface fluctuations and their consequences on the local (re)organization of the suspension. It is shown that particles experience a significant effective interaction in the vicinity of the interface. This potential of mean force is always attractive, with range controlled by the surface correlation length. We suggest that, under some circumstances, surface-induced attraction may have a strong influence on the local particle distribution.

PACS numbers: 05.20.-y,82.70.-y,87.16.Dg
\end{abstract}

Colloidal suspensions are solutions of fairly large objects, with typical size ranging from $1 \mathrm{~nm}$ to $1 \mu \mathrm{m}$. The primary question of their stability and phase behaviour is the foundation of many technological applications [1]. Formally, the statistical description of a colloidal dispersion involves colloid-colloid, colloid-solvent and solventsolvent interactions. However, such detailed and complex information is usually not required to understand essential features, and it has been found more appropriate to develop effective descriptions where the colloids interact through a potential of mean force 2]. The individual forces acting between particles then depend explicitly on the temperature and on the chemical potential of the solvent. Examples of such effective potentials include dispersion forces, DLVO theory for charged systems, or depletion interactions in polydispersed solutions.

On the other hand, it has been recognized long ago that surface effects are prevailing in confined colloidal systems [3, 4]. The mutual influence of bulk and surface properties on each other is a challenging problem, that conversely may lead to unusual behaviours. For instance, when a bidispersed hard-sphere suspension is brought in contact with a flat substrate, excluded-volume effects are known to push the larger beads toward the wall of the sample [5]. Recent experiments done with curved or corrugated surfaces have shown that geometric features of the surface can also create and modulate entropic force fields [6]. These depletion forces can be used to grow oriented colloidal crystal, with numerous potential applications such as the fabrication of photonic bandgap crystals [7].

In this Letter, we present some new findings regarding the static organization of nanoparticles near a fluctuating surface. Adsorption of colloidal particles on a flexible interface is an essential step in many biological processes, and the underlying physics of this mecha-

*Electronic adress: th.bickel@cpmoh.u-bordeaux1.fr nism has been extensively studied for simple model systems $8,[9,10,11]$. Thermal undulations of the surface are nevertheless disregarded in most theoretical works, even though they are expected to locally alter the particle distribution 12, 13]. Technically, the difficulty lies in the interplay between bulk and surface degrees of freedom. This question is considered hereafter, with particular emphasis given to indirect interactions between colloids that merge from surface fluctuations. Our aim is to bring down the complexity of the problem and, ultimately, to come down to an effective description for the colloidal suspension.

The remaining of the presentation proceeds as follows. First, we derive general expressions for the one and twobody potentials. We then express the local density profile and the corresponding adsorbance. The computation of the effective pair-potential for relevant experimental configurations is presented in the next section. Finally, we draw some concluding remarks on whether surface fluctuations could induce colloid crystallization.

The physical system under consideration is a dilute colloidal suspension in contact with a fluid interface, defined here as a sharp boundary. The results derived in this article are intended to be as general as to describe a wide class of soft surfaces, ranging from liquid-liquid interfaces to surfactant monolayers or bilayers. We assume that the surface deviates only slightly from the horizontal plane. In the Monge representation, a point of the corrugated surface has coordinates $(x, y, h(x, y))$. The height function $h(x, y)$ may take either positive or negative values. In what follows, we shall use the notation $\boldsymbol{r}=(\boldsymbol{\rho}, z)$, where $\boldsymbol{\rho}=(x, y)$ is the transverse vector and $z$ the perpendicular distance. The total Hamiltonian $\mathcal{H}$ of the system can be written as the sum of three terms

$$
\mathcal{H}=\mathcal{H}_{m}+\mathcal{H}_{c c}+\mathcal{H}_{c m} .
$$

The first contribution is the energy of the weakly curved surface 14]

$$
\mathcal{H}_{m}[h]=\frac{1}{2} \int d^{2} \boldsymbol{\rho}\left[\kappa(\Delta h)^{2}+\sigma(\nabla h)^{2}+\mu h^{2}\right] .
$$


The parameter $\mu$, the bending rigidity $\kappa$ and the surface tension $\sigma$ are the bare elastic constants of the interface, in the absence of particle. It is convinient to define the expectation value $\langle X\rangle_{0}$ of a given functional $X[h]$ as

$$
\langle X\rangle_{0}=\frac{1}{\mathcal{Z}_{0}} \int \mathcal{D} h X[h] e^{-\beta \mathcal{H}_{m}[h]},
$$

with $\mathcal{Z}_{0}=\int \mathcal{D} h \exp \left[-\beta \mathcal{H}_{m}[h]\right]$ the partition function of the interface. We adopt the usual notation $\beta=1 / k_{B} T$, with $T$ the absolute temperature and $k_{B}$ the Boltzmann constant. We also define the height correlation function - or Green function - of the surface

$$
G\left(\boldsymbol{\rho}-\boldsymbol{\rho}^{\prime}\right)=\left\langle h(\boldsymbol{\rho}) h\left(\boldsymbol{\rho}^{\prime}\right)\right\rangle_{0}-\langle h(\boldsymbol{\rho})\rangle_{0}\left\langle h\left(\boldsymbol{\rho}^{\prime}\right)\right\rangle_{0},
$$

from which we extract the mean-squared displacement $\xi_{\perp}^{2}=G(0)$.

The direct colloid-colloid interaction $\mathcal{H}_{c c}$ does not need to be specified at this point. The last term in eq. (11), $\mathcal{H}_{c m}$, represents the colloid-interface interaction. It is generally a complicated function of particle position and surface configurations. However, since we are interested in the regime where the interface undergoes strong fluctuations, we restrict the discussion to colloids with size much smaller than $\xi_{\perp}$. Typically, the particles under consideration have diameter of a few tens of nanometers, whereas the surface roughness lies in the micrometer range. This assumption allows us to neglect finite-size effects and to select a local potential, that depends only on the relative perpendicular distance between the colloid and the surface. Despite this simplification, $\mathcal{H}_{c m}$ is still expected to be quite complicated. We next assume that the surface potential is "short-ranged" (in comparison to the length scale $\xi_{\perp}$ ), with typical extension of the order of the colloidal size. We then choose the following contact potential

$$
\beta \mathcal{H}_{c m}[h]=-\frac{\omega}{2} \sum_{i=1}^{N} \delta\left(z_{i}-h\left(\boldsymbol{\rho}_{i}\right)\right),
$$

with $\delta$ the Dirac distribution. In this definition, the discrete sum runs over all particles with position $\boldsymbol{r}_{i}=$ $\left(\boldsymbol{\rho}_{i}, z_{i}\right)$, and $\omega>0$ is the (surface) coupling constant. Actually, $\omega$ plays the role of an extrapolation length as usually encountered in surface critical phenomena 15]. In this model, the surface is penetrable and the colloids can accomodate on both sides of the interface. It is clear that the potential defined in eq. (5) is rather different from usual DLVO or hydratation potentials. Nevertheless, we focus in the following on colloids that are slightly bound to the surface, so that we do not expect the microscopic details of the potential to be pertinent. If necesseray, the strength of the attraction $\omega$ may be related to the depth $U_{0}$ and the range $b$ of a more realistic potential through the relation $k_{B} T \omega=U_{0} b$.

To derive the statistical quantities of interest, we first evaluate the grand canonical partition function

$$
\mathcal{Z}_{G}=\sum_{N=0}^{+\infty} \frac{f^{N}}{\lambda^{3 N} N !} \int \prod_{i=1}^{N} d \boldsymbol{r}_{i} \int \mathcal{D} h e^{-\beta \mathcal{H}[h]},
$$

whith $\lambda$ the thermal wavelength of the particles under consideration, and $f$ the fugacity. The functional integral extends over all conformations of the field $h(x, y)$. The contribution $N=0$ is the partition function $\mathcal{Z}_{0}$ of an interface in a particle-free environment. The term $N=1$ corresponds to an interface interacting with one colloid, and so on. What makes the evaluation of eq. (6) difficult is the fact that bulk and surface degrees of freedom are coupled through $\mathcal{H}_{c m}$. However, for the Gaussian theory considered here, surface undulations can be traced out by using the standard cumulant method [16]. After some algebra, we find that the colloids interact through an effective potential $\mathcal{H}_{c c}^{\text {eff }}=\mathcal{H}_{c c}+\mathcal{U}$. The potential of mean force $\mathcal{U}$ involves many-body interactions

$$
\begin{aligned}
\mathcal{U}\left(\boldsymbol{r}_{1}, \ldots, \boldsymbol{r}_{N}\right) & =-k_{B} T \ln \left\langle e^{-\beta \mathcal{H}_{c m}[h]}\right\rangle_{0} \\
& =\sum_{i=1}^{N} \mathcal{U}_{1}\left(\boldsymbol{r}_{i}\right)+\sum_{\{i, j\}} \mathcal{U}_{2}\left(\boldsymbol{r}_{i}, \boldsymbol{r}_{j}\right)+\ldots .
\end{aligned}
$$

As we are only interested in the weak adsorption regime, contributions from three-body terms and beyond can be neglected if $\omega$ is small enough. The one and two-body potentials are respectively

$$
\begin{aligned}
& \beta \mathcal{U}_{1}(\boldsymbol{r})=-\frac{\omega}{2} \Phi_{1}(z), \text { and } \\
& \beta \mathcal{U}_{2}\left(\boldsymbol{r}, \boldsymbol{r}^{\prime}\right)=-\frac{\omega^{2}}{8}\left[\Phi_{2}\left(\boldsymbol{\rho}, \boldsymbol{\rho}^{\prime} ; z, z^{\prime}\right)-\Phi_{1}(z) \Phi_{1}\left(z^{\prime}\right)\right]
\end{aligned}
$$

where we have introduced the useful function $\Phi_{N}\left(\boldsymbol{r}_{1}, \ldots, \boldsymbol{r}_{N}\right)=\left\langle\prod_{i=1}^{N} \delta\left(z_{i}-h\left(\boldsymbol{\rho}_{i}\right)\right)\right\rangle_{0}$. The latter can be computed exactly [13], and we simply sketch the results

$$
\Phi_{1}(z)=\frac{1}{\sqrt{2 \pi} \xi_{\perp}} \exp \left[-\frac{z^{2}}{2 \xi_{\perp}^{2}}\right]
$$

for $N=1$, and

$$
\begin{aligned}
& \Phi_{2}\left(\boldsymbol{\rho}, \boldsymbol{\rho}^{\prime} ; z, z^{\prime}\right)=(2 \pi)^{-1}[\operatorname{det} \mathcal{G}]^{-1 / 2} \\
& \quad \times \exp \left[-\frac{G(0)}{2 \operatorname{det} \mathcal{G}}\left(z^{2}+z^{\prime 2}\right)+\frac{G\left(\boldsymbol{\rho}-\boldsymbol{\rho}^{\prime}\right)}{\operatorname{det} \mathcal{G}} z z^{\prime}\right],
\end{aligned}
$$

for $N=2$, where we $\operatorname{define} \operatorname{det} \mathcal{G}=G^{2}(0)-G^{2}\left(\boldsymbol{\rho}-\boldsymbol{\rho}^{\prime}\right)$. Note that eq. (8)-10) provide an explicit criterion for the validity of the cumulant approximation. Indeed, the dimensionless coupling constant $\omega / \xi_{\perp}$ has to be identified as the "small" parameter of the cumulant expansion. The relevance of our approach is then ensured as long as $\omega / \xi_{\perp}<1$.

Let us now discuss some plausible outcomes of our analysis. The density profile can be estimated from the approximate relation $\rho(z)=\rho_{\infty} \exp \left[-\beta \mathcal{U}_{1}\right]$, with $\rho_{\infty}=f / \lambda^{3}$ the bulk value [17]. Results from the last section yield

$$
\rho(z)=\rho_{\infty} \exp \left[\frac{\omega}{\sqrt{8 \pi} \xi_{\perp}} \exp \left[-\frac{z^{2}}{2 \xi_{\perp}^{2}}\right]\right] .
$$




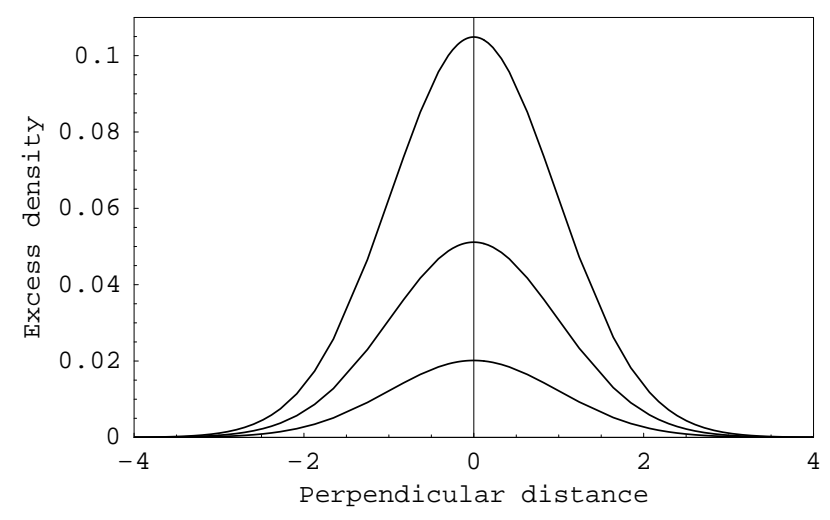

FIG. 1: Excess particle density $\left(\rho / \rho_{\infty}-1\right)$ as a function of the (dimensionless) elevation $z / \xi_{\perp}$, for different values of the surface coupling constants $\omega / \xi_{\perp}=0.1,0.25$ and 0.5 , respectively.

The variation of concentration upon distance is represented in Fig. (1). Remarkably, the density reaches its bulk value over a distance that is not the extrapolation length of the surface potential. Instead, this range is set by the elastic properties of the fluctuating interface via the roughness $\xi_{\perp}$. The potential $\mathcal{U}_{1}$ can be interpreted as a "condensation" potential forcing the colloids to be localized in the vicinity of the surface $z=0$. The excess of particles is further characterized by the adsorbance

$$
\Gamma=\int_{-\infty}^{+\infty}\left[\rho(z)-\rho_{\infty}\right] d z=\frac{1}{2} \omega \rho_{\infty}+\mathcal{O}\left(\omega^{2}\right)
$$

As expected, the adsorbance $\Gamma$ is positive - denoting particle accumulation - and scales as the coupling constant $\omega$. Note that in the case of colloid-surface repulsion $(\omega<0)$, colloids are depleted from the interface and the corresponding adsorbance is negative (see also ref. [13]). Finally, let us comment on the limit of an infinitely rigid interface, corresponding to $\kappa \gg k_{B} T$. From the representation of the $\delta$-distribution $\delta(x)=\lim _{\varepsilon \rightarrow 0}\left(2 \pi \varepsilon^{2}\right)^{-1} \exp \left[-x^{2} /\left(2 \varepsilon^{2}\right)\right]$, we immediately recover the anticipated Boltzmann distribution $\rho(z)=$ $\rho_{\infty} \exp \left[-\beta \mathcal{H}_{c m}[0](z)\right]$, with the surface potential given in eq. (5).

We now focus on the two-body potential. From the general property of random variable theory according to which $\langle X Y\rangle-\langle X\rangle\langle Y\rangle \geq 0$, one can directly infer that $\Phi_{2}\left(\boldsymbol{\rho}, \boldsymbol{\rho}^{\prime} ; z, z^{\prime}\right) \geq \Phi_{1}(z) \Phi_{1}\left(z^{\prime}\right)$. Comparing with Eq. (8) allows us to conclude that $\mathcal{U}_{2}$ is always attractive, whatever the sign of $\omega$. Indeed, surface entropy is found to increase in every instance when particles are getting closer, leading to fluctuation-induced attraction.

The two-body potential is shown in Fig. (2) for a fluid membrane $(\sigma=0)$. For fixed perpendicular distances $z$ and $z^{\prime}, \mathcal{U}_{2}$ vanishes as the relative parallel distance $l=\left|\boldsymbol{\rho}-\boldsymbol{\rho}^{\prime}\right|$ goes to infinity. When the latter is fixed, the interaction dies off very rapidely as $z$ or $z^{\prime} \gg \xi_{\perp}$. From Eq. (8)-(10), it can be noticed that $\mathcal{U}_{2}$ is actually boundless when $l \rightarrow 0$. For two particles at the same elevation $z=z^{\prime}=0$ we find

$$
\beta \mathcal{U}_{2}(l) \sim-\frac{1}{16 \pi}\left(\frac{\omega}{\xi_{\perp}}\right)^{2}\left(1-\frac{G(l)^{2}}{G(0)^{2}}\right)^{-1 / 2},
$$

where the Green function $G(l)$ depends on the elastic properties of the surface under consideration. As a matter of fact, this behaviour is reminiscent of the shortrange colloid-surface potential. In a real system, however, particles always have a finite size so that a minimal distance between colloids is set by their diameter. Moreover, because $G(l)$ usually involves logarithmic contributions at short distances, the attraction is expected to increase only very slowly. It thus remains moderate even at low separations.

So far, we have not specified the nature of the surface and of the inter-particle potential $\mathcal{H}_{c c}$. To keep the discussion simple, we describe the colloids as hard spheres of diameter $d$. We consider as a first example an interface between two immiscible liquids, with no bending rigidity. The height correlation function is $G(l)=(2 \pi \beta \sigma)^{-1} \mathrm{~K}_{0}(l / \xi)$, with $\xi=(\sigma / \mu)^{1 / 2}$ the capillary length. At short distances, the Bessel function $\mathrm{K}_{0}$ behaves like $\mathrm{K}_{0}(x) \sim_{0}-\ln (x)$ and the mean square fluctuations of the interface involves some molecular size $\lambda_{c}$ 14]. With $\lambda_{c} / \xi=10^{-4}, d / \xi=10^{-3}$, and $\omega / \xi_{\perp}=0.5$, we obtain the value at contact $\mathcal{U}_{2}(d) \approx 5 \times 10^{-3} k_{B} T$.

In the opposite limit of a fluid membrane with vanishing surface tension $\sigma=0$, the height fluctuations are much larger and surface-mediated attraction is expected to be stronger. The short-distances behaviour of the propagator that appears in eq. (10) is

$$
G(l) \simeq G(0)\left(1+\frac{2}{\pi} \frac{l^{2}}{\xi_{\|}^{2}} \ln \left(\frac{l}{\xi_{\|}}\right)\right) \text {for } \quad l \ll \xi_{\|},
$$

with $\xi_{\|}=(4 \kappa / \mu)^{1 / 4}$ the in-plane correlation length of the membrane. From eq. (13) we obtain the potential "at contact"

$$
\beta \mathcal{U}_{2}\left(\left|\boldsymbol{r}-\boldsymbol{r}^{\prime}\right|=d\right) \approx-\frac{1}{32 \sqrt{\pi}}\left(\frac{\omega}{\xi_{\perp}}\right)^{2}\left[\frac{d^{2}}{\xi_{\|}^{2}} \ln \left(\frac{\xi_{\|}}{d}\right)\right]^{-1 / 2}
$$

For sensible values of the parameters $d / \xi_{\|}=10^{-3}$ and $\omega / \xi_{\perp}=0.5$, the depth of the potential well is comparable to the thermal energy: $\mathcal{U}_{2}(d) \approx 1.7 k_{B} T$. Actually, the strength of the interaction increases as the ratio $d / \xi_{\|}$decreases. The only requirement limiting the lower bound of this ratio is that we have to remain in the colloidal domain, i.e. the diameter of the colloids has to be large compared to the size of the solvent molecules.

The original motivation of our work was to describe the statistical properties of colloidal suspension in contact with a soft surface. The main outcome of this article is that, as a result of surface fluctuations, particles experience a potential of mean force $\mathcal{U}=\mathcal{U}_{1}+\mathcal{U}_{2}+\ldots$, that 


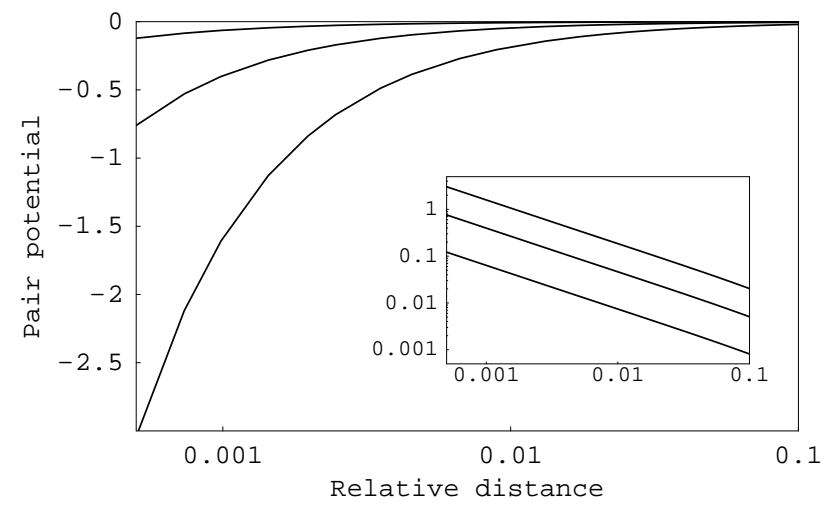

FIG. 2: Effective two-body potential (in units of $k_{B} T$ ) as a function of the relative distance $l / \xi_{\|}$, for a fluid membrane $(\sigma=0)$, and for two colloids at the same elevation $z=z^{\prime}=0$. Note the logarithmic scale on the horizontal axis. The different curves correspond to surface coupling constants $\omega / \xi_{\perp}=0.1,0.25$ and 0.5 , respectively. In the inset, we show $\ln \left|\beta \mathcal{U}_{2}\right|$ as a function of $\ln \left(l / \xi_{\|}\right)$, illustrating eq. (15).

is not pairwise additive. Indeed, the expectation value eq. (7) involves three-body contributions and higher, but those are neglectable at low density or for small surface coupling parameter. The leading contribution, $\mathcal{U}_{1}$, act as an external field that condensates the colloids in the vicinity of the interface. In the weak adsorption regime, particle accumulation remains moderate: for $\omega / \xi_{\perp}=0.5$, the density at elevation $z=0$ is only $10 \%$ higher than its bulk value. But once localization is enforced, particles attract each other through the pair potential $\mathcal{U}_{2}$ whose strength can be quite substantial. Under some circumstances, it is of the order of the thermal energy $k_{B} T$. Accordingly, these forces may have a strong influence on the local organization of the suspension, and surface fluctuations might even possibly promote the nucleation of an ordered phase of colloids in the vicinity of the interface. This suggestion would clearly deserve more attention as it may provide a new route for directed self-assembly of mesoscopic colloidal structures.

Finally, we note that the mechanism leading to particle attraction does not seem to depend crucially on the exact nature of $\mathcal{H}_{c m}$. The effective potential is solely driven by the entropy of the surface and shares many similarities with the well-known depletion forces. In particular, the dependency of $\mathcal{U}_{2}$ on the elastic parameters $\kappa, \sigma$ and $\mu$ turns out to be a great advantage as it may allow to tune the interaction. This can be achieved for instance by adding cosurfactant molecules to monolayers and bilayers, or by slightly changing the temperature for an interface between two immiscible liquids near the mixing transition. Indeed, the surface tension scales in the latter situation as $\sigma \sim\left|T-T_{c}\right|^{-\mu}$, with $\mu>0$ a critical exponant, in such way that the fluctuations amplitude is extremely sensitive to a minute variation of temperature. It should therefore be possible, in principle, to control the effective interactions between colloids.
[1] W.B. Russel, D.A. Saville, and W.R. Schowalter, Colloidal Dispersions (Cambridge University Press, 1989).

[2] C.N. Likos, Phys. Rep. 3482001267.

[3] S. Asakura and F. Oosawa, J. Chem. Phys. 22 (1954) 1255.

[4] J.-F. Joanny, L. Leibler, and P.-G. de Gennes, J. Polym. Sci. 17 (1979) 1073.

[5] P.D. Kaplan, J.L. Rouke, A.G. Yodh, and D.J. Pine, Phys. Rev. Lett. 72 (1994) 582.

[6] A.D. Dinsmore, A.G. Yodh, and D.J. Pine, Nature (London) 383 (1996) 239.

[7] K.-H. Lin, J.C. Crocker, V. Prasad, A. Schofield, D.A. Weitz, T.C. Lubensky, and A.G. Yodh, Phys. Rev. Lett. 85 (2000) 1770.

[8] R. Lipowsky and H.-G. Döbereiner, Europhys. Lett. 43 (1998) 219.

[9] F. Divet, T. Biben, I. Cantat, A. Stephanou, B. Fourcade, and C. Misbah C., Europhys. Lett. 60 (2002) 795.

[10] M. Deserno and T. Bickel, Europhys. Lett. 62 (2003) 767.
[11] D. van Effenterre and D. Roux, Europhys. Lett. 64 (2004) 543.

[12] T. Bickel, J. Chem. Phys. 118 (2003) 8960.

[13] T. Bickel, M. Benhamou, and H. Kä̈di, Phys. Rev. E 70 (2004) 05xxxx.

[14] S. Safran, Statistical Thermodynamics of Surfaces, Interfaces and Membranes (Addison-Wesley, Reading, MA, 1994).

[15] K. Binder, Phase Transitions and Critical Phenomena, Vol.8, edited by C. Domb and J.L. Lebowitz (Academic Press, London, 1983).

[16] C. Itzykson and J.-M. Drouffe, Statistical Field Theory, Vol.1 (Cambridge University Press, 1989).

[17] A more refined evaluation of $\rho(z)=\left\langle\sum_{i=1}^{N} \delta\left(\mathbf{r}-\mathbf{r}_{i}\right)\right\rangle$ can be performed by applying a suitable renormalization scheme, and gives essentially the same result at first order (unpublished results). 